\title{
IgA anti-tissue transglutaminase antibodies and IgG antibodies against deamidated gliadin peptides as predictors of celiac disease
}

\author{
Gonzalo Ortiz, M.D. ${ }^{a}$, Gabriela Messere, M.D. ${ }^{a}$, María del C. Toca, M.D. ${ }^{a}$, Mirian Fiorucci, M.D. ${ }^{b}$, \\ Román Bigliardi, M.D. ${ }^{a}$, Jorge Vidal, M.D. ${ }^{a}$ and Ricardo Reynoso, M.D. ${ }^{a}$
}

\begin{abstract}
Objective. To compare the performance of IgA anti-tissue transglutaminase antibodies (IgA anti-tTG), IgA anti-endomysial antibodies (IgA EMA), and $\operatorname{IgA} / \operatorname{IgG}$ antibodies against deamidated gliadin peptides (IgA/IgG anti-DGP) for the diagnosis of celiac disease.

Methods. Descriptive study in patients with celiac disease. Anti-DGP (IgA/IgG), IgA EMA, IgA anti-tTG antibodies were measured and an intestinal biopsy was done. Sex: female $(61 \%)$. Median age: 78.4 months old.

Results. A total of 136 children were included; 108 had high IgA anti-DGP titers; 124, increased IgG anti-DGP titers; 128, positive IgA EMA titers; and 130, increased IgA anti-tTG titers. High IgG anti-DGP titers were observed in $4 / 6$ patients with negative IgA anti-tTG antibodies. The combination of IgG antiDGP + IgA anti-tTG antibodies showed a positive correlation in 134 patients and the IgG anti-DGP + EMA combination was positive in 133 children.

Conclusion. IgA EMA, IgA anti-tTG, and IgG anti-DGP antibodies exhibited an adequate specificity and sensitivity. The IgG anti-DGP/anti-tTG combination showed a 98-99 \% sensitivity and a $100 \%$ specificity. The anti-tTG and IgG anti-DGP option yields excellent results, with a low cost and independence from the observer.

Key words: celiac disease, antibodies, diagnosis, children.
\end{abstract}

http: / / dx.doi.org/10.5546/ aap.2019.eng.52

To cite: Ortiz G, Messere G, Toca MC, Fiorucci M, et al. IgA anti-tissue transglutaminase antibodies and IgG antibodies against deamidated gliadin peptides as predictors of celiac disease. Arch Argent Pediatr 2019;117(1):52-55.

a. Division of Pediatric Gastroenterology, Hepatology and Nutrition, Department of Pediatrics.

b. Laboratory Service. Hospital Nacional "Alejandro Posadas." Province of Buenos Aires.

E-mail address:

Gonzalo Ortiz, M.D.: ortizgonzalojavier@gmail.com

Funding: None.

Conflict of interest: None.

Received: 9-6-2017

Accepted: 8-15-2018

\section{INTRODUCTION}

Celiac disease (CD) is an immune-mediated, chronic systemic disease triggered by the intake of gluten-containing grains, which affects the small intestine of genetically predisposed individuals. ${ }^{1}$

The antibodies against one or more of the individual's own proteins (autoantibodies) are markers of autoimmunity; they may be anti-tissue transglutaminase (anti-tTG) or anti-endomysial (EMA) autoantibodies. ${ }^{2-4}$

The EMA antibody is the most specific test. It exhibits a high sensitivity (90-98\%) and specificity; it is done by indirect immunofluorescence (IIF) and is observer-dependent, so the diagnostic algorithm is used as a confirmatory test.

The anti-tTG antibody test is done using an enzyme-linked immunosorbent assay (ELISA), which is more objective and simple and has been recommended as the initial tool for the diagnostic algorithm of CD. ${ }^{2-4}$

It has been demonstrated that the sensitivity of the anti-tTG antibody reduces in patients with mild intestinal damage, which is frequent in individuals with a low gluten intake and in family members of celiac patients. Another population that may present false negative results when using autoantibodies (anti-tTG and EMA) are children younger than 3 years, who have a low autoimmune response. ${ }^{2,3}$ In this group of patients, the combination of anti-tTG antibodies and IgG antibodies against deamidated gliadin peptides (IgG anti-DGP) may improve the detection of CD. . $^{2-5}$

The tests used to measure IgG anti-DGP antibodies improve the effectiveness of diagnosis by increasing sensitivity while maintaining specificity. ${ }^{6}$ The intestinal biopsy is still an indisputable diagnostic criterion for $\mathrm{CD} .6,7$

The objective of our study was to compare the performance of IgA anti-tTG, IgA EMA, and IgA/ IgG anti-DGP antibodies for $\mathrm{CD}$ diagnosis.

\section{MATERIAL AND METHODS}

This was a descriptive and observational study conducted between January 2011 and December 2014. Patients who attended the Division of 
Pediatric Gastroenterology of the Department of Pediatrics of Hospital Nacional "Alejandro Posadas" for an esophagogastroduodenoscopy (EGD) due to suspicion of CD were included.

All children had total IgA and serological tests: IgA/IgG anti-DGP using the ELISA (QUANTA Lite (eliac), with a cut-off value > 20 IU; IgA EMA using the IIF method, considering as positive a dilution value > 1:5; and IgA anti-tTG using the ELISA (QUANTA), with a cut-off value $>20 \mathrm{U} / \mathrm{L}$.

All patients had an EGD; 6 biopsies were done (4 in the second part of the duodenum and 2 in the duodenal bulb).

Histopathological results were described using the Marsh-Oberhuber classification: Marsh 0: normal mucous membrane; Marsh 1: increased intra-epithelial lymphocytes (IELs); Marsh 2: increased IELs with crypt hyperplasia; and Marsh 3: atrophic villi. Patients were considered to have CD if they were classified as Marsh 2 and 3.

The protocol was assessed and approved by the Ethics Committee of Hospital Nacional "Alejandro Posadas." All parents or legal guardians were asked to give their informed consent, while children older than 12 years gave their assent.

Statistical data: The sensitivity, specificity, positive predictive value (PPV), and negative predictive value (NPV) of IgA/IgG anti-DGP, IgA EMA and IgA anti-tTG antibodies were measured, as well as the IgG anti-DGP/IgA antitTG and IgA EMA/IgG anti-DGP combinations.

All measurements were done using a $95 \%$ confidence interval. The $\chi^{2}$ test and receiver operating characteristic (ROC) curve with a $95 \%$ confidence interval were used.

\section{RESULTS}

During the four-year study period, 136 children were included, 83 (61\%) of which were girls and $53(39 \%)$, boys. Patients' median age was 78.4 months (range: 12-192 months). No child had total IgA deficiency.

The most common clinical presentation was classic in 83 children $(61 \%)$, followed by the atypical presentation in 36 children $(26 \%)$; the remaining 17 patients $(13 \%)$ were asymptomatic. More than one positive antibody was observed in the latter group.

The duodenal biopsy was confirmatory for CD in all 136 children. Two patients were classified as Marsh 2, and 134, as Marsh 3 (102: 3c; 29: 3b; and 3: 3a).

The serological tests showed a specificity of $100 \%$ for IgA EMA and IgA anti-tTG antibodies, and of $97 \%$ for IgG anti-DGP antibodies. Sensitivity was high for the IgA EMA and anti-tTG tests (94-96 \%, respectively), moderate for the IgG anti-DGP test $(91 \%)$, and low for the IgA anti-DGP test $(79 \%)$. See Table 1.

It is worth noting that, the combination of two antibodies (IgG anti-DGP + IgA EMA and / or IgG anti-DGP + IgA anti-tTG) improved sensitivity to 98-99 \% and specificity to $100 \%$. See Table 2.

In the population of children younger than 3 years ( $n: 39)$, serological tests had a low sensitivity; it was $90 \%$ for IgA anti-tTG antibodies. The 4 patients with normal antitTG had high IgG anti-DGP titers. All of them presented with the typical symptoms. See Table 3.

\section{DISCUSSION}

The most sensitive and specific tests are those capable of detecting anti-tTG and EMA autoantibodies with a 90-98 \% sensitivity and a 95-98\% specificity in patients with untreated CD, with symptoms and severe mucosal damage. ${ }^{8}$

In recent years, ELISA tests have been recommended because they measure anti-tTG and anti-DGP antibodies, so as to improve sensitivity.

TABLEA 1. Sensitivity and specificity of serological tests

\begin{tabular}{|c|c|c|c|c|}
\hline Patients & IgA anti-DGP & IgG anti-DGP & IgA EMA & IgA anti-tTG \\
\hline Celiac patients:: 136 & $108(+) / 28(-)$ & $124(+) / 12(-)$ & $128(+) / 8(-)$ & $130(+) / 6(-)$ \\
\hline Sensitivity & $79 \%$ & $91 \%$ & $94 \%$ & $96 \%$ \\
\hline Specificity & $97 \%$ & $97 \%$ & $100 \%$ & $100 \%$ \\
\hline PPV & $98 \%$ & $98 \%$ & $100 \%$ & $100 \%$ \\
\hline NPV & $67 \%$ & $82 \%$ & $87 \%$ & $90 \%$ \\
\hline
\end{tabular}

DGP: deamidated gliadin peptide; EMA: anti-endomysial antibody; anti-tTG: anti-tissue transglutaminase antibody; PPV: positive predictive value; NPV: negative predictive value; IgA: immunoglobulin A; IgG: immunoglobulin G. 
Both IgA/IgG anti-DGP antibodies demonstrated a better sensitivity than anti-gliadin (classic) antibodies, especially IgG antibodies, which have a better specificity in pediatric patients and are useful in patients with IgA deficiency. ${ }^{7-9}$

In this study, IgG anti-DGP, anti-tTG, and EMA showed a similar sensitivity $(91 \%, 96 \%$, and $94 \%$, respectively), whereas IgA anti-DGP had a lower sensitivity of $71 \%$. Two antibodies were combined in order to improve their performance: with EMA/ IgG anti-DGP, sensitivity improved to $98 \%$ and had a NPV of $95 \%$; with anti-tTG/IgG anti-DGP, sensitivity improved even more, to $99 \%$, with a NPV of $97 \%$.

The analysis of the results that reduced serological test sensitivity (false negative results) showed that, when considering the age of patients with suspected $C D, 5$ out of the 8 patients who had negative EMA antibodies and all 6 patients with normal anti-tTG antibodies were children younger than 3 years. The combination of any of these with IgG anti-DGP improved the sensitivity for $\mathrm{CD}$ diagnosis in this age group.

Specificity was $100 \%$ with a $100 \%$ PPV for EMA and anti-tTG antibodies, and $97 \%$ with a $98 \%$ PPV for IgA/IgG anti-DGP antibodies. The specificity of the IgG anti-DGP antibody improved remarkably compared to that observed in previous studies with the classical IgG antigliadin antibody: $97 \%$ versus $63 \%$. The EMA/ IgG anti-DGP or anti-tTG/IgG anti-DGP combinations showed a high specificity, which was, in both cases, $100 \%$ with a $100 \%$ PPV.

In our study, IgG anti-DGP antibodies exhibited a higher sensitivity and specificity than IgA antibodies. As detailed by Prause et al., IgG anti-DGP antibodies had a better performance than IgA antibodies, especially in patients with IgA deficiency and younger than 2 years. In the first years of life, tTG may show false negative results or marked fluctuations because its immunity development mechanism may be slower, with high titers only 1-2 years after exposure to gluten. ${ }^{10-12}$

During our study period, no patient had IgA deficiency, but several publications have shown that the IgG anti-DGP antibody has an adequate sensitivity and specificity in this risk population, which makes it suitable for $\mathrm{CD}$ diagnosis. ${ }^{10-12}$

\section{CONCLUSIONS}

Our study confirmed the adequate specificity and sensitivity of the tests to detect EMA, antitTG, and IgG anti-DGP antibodies, and the low sensitivity of the IgA anti-DGP test. The combination of the IgG anti-DGP serological

TABLE 2. Sensitivity and specificity of combined serological tests

\begin{tabular}{lcc}
\hline Patients & IgA EMA + IgG anti-DGP & IgA anti-tTG + IgG anti-DGP \\
\hline Celiac patients: 136 & $133(+) / 3(-)$ & $134(+) / 2(-)$ \\
Sensitivity & $98 \%$ & $99 \%$ \\
Specificity & $100 \%$ & $100 \%$ \\
PPV & $100 \%$ & $100 \%$ \\
NPV & $95 \%$ & $97 \%$ \\
\hline
\end{tabular}

DGP: deamidated gliadin peptide; EMA: anti-endomysial antibody; anti-tTG: anti-tissue transglutaminase antibody; PPV: positive predictive value; NPV: negative predictive value; IgA: immunoglobulin A; IgG: immunoglobulin G.

TABLE 3. Sensitivity and specificity of serological tests in patients younger than 3 years

\begin{tabular}{lcccc} 
Younger than 3 years (n: 39) & Sensitivity & Specificity & PPV & NPV \\
\hline IgA anti-DGP (n: 29) & $74 \%$ & $100 \%$ & $100 \%$ & $55 \%$ \\
IgG anti-DGP (n: 33) & $85 \%$ & $100 \%$ & $100 \%$ & $67 \%$ \\
IgA EMA (n: 34) & $87 \%$ & $100 \%$ & $100 \%$ & $68 \%$ \\
IgA anti-tTG (n: 35) & $90 \%$ & $100 \%$ & $100 \%$ & $75 \%$ \\
\hline
\end{tabular}

DGP: deamidated gliadin peptide; EMA: anti-endomysial antibody; anti-tTG: anti-tissue transglutaminase antibody; IgA: immunoglobulin A; IgG: immunoglobulin G; PPV: positive predictive value; NPV: negative predictive value. 
test with EMA and anti-tTG showed a 98-99\% sensitivity and a $100 \%$ specificity. The anti-tTG and IgG anti-DGP option yields excellent results, with a low cost and independence from the observer.

\section{REFERENCES}

1. Ludvigsson JF, Leffler DA, Bai JC, Biagi F, et al. The Oslo definitions for coeliac disease and related terms. Gut. 2013; 62(1):43-52.

2. Fasano A, Araya M, Bhatnagar S, Cameron D, et. al. Federation of International Societies of Pediatric Gastroenterology, Hepatology, and Nutrition Consensus Report on Celiac Disease. J Pediatr Gastroenterol Nutr. 2008; 47(2):214-9.

3. Aleanzi M, Demonte A, Esper C, Garcilazo S, et.al. Celiac Disease: Antibody recognition against native and selectively deaminated gliadin peptides. Clin Chem. 2001; 47(11):2013-28.

4. Murch S, Jenkins $H$, Auth $M$, Bremmer R, et al. Joint BSPGHAN and Coeliac UK guidelines for the diagnosis and management of coeliac disease in children. Arch Dis Child. 2013; 98(10):806-11.

5. Kupfer S, Jabri B. Pathophysiology of celiac disease. Gastrointest Endosc Clin N Am. 2012; 22(4):639-60.
6. Volta U. Coeliac Disease: Time for a new diagnostic approach in symptomatic children. J Pediatr Gastroenterol Nutr. 2013; 56(3):241.

7. Rubio-Tapia A, Hill ID, Kelly CP, Calderwood A, et al. ACG clinical guidelines: diagnosis and management of celiac disease. Am J Gastroenterol. 2013;108(5):656-76.

8. NiveloniS, Sugai E, Cabanne A, VazquezH, etal. Antibodies against synthetic deamidate gliadin peptides as predictors of celiac disease: Prospective assessment in an adult population with a high pretest probability of disease. Clin Chem. 2007; 53(12):2186-92.

9. Vermeersch P, Geboes K, Mariën G, Hoffman I, et al. Serological diagnosis of celiac disease: Comparative analysis of different strategies. Clin Chim Acta. 2012;413(2122):1761-7.

10. Giersiepen K, Lelgemann M, Stuhldreher N, Ronfani L, et al. Accuracy of Diagnostic Antibody Tests for Coeliac Disease in Children: Summary of an Evidence Report. J Pediatr Gastroenterol Nutr. 2012; 54(2):229-41.

11. Husby S, Koletzko S, Korponay-Szabó IR, Mearin ML, et al. European Society for Pediatric Gastroenterology, Hepatology, and Nutrition guidelines for the diagnosis of coeliac disease. J Pediatr Gastroenterol Nutr. 2012; 54(1): 136-60.

12. Bufler G, Heilig G, Ossiander F, Freudenberg V, et al. Diagnostic performance of three serologic tests in childhood celiac disease. Z Gastroenterol. 2015; 53(2):108-14. 